\title{
Hydrodynamic Transport Coefficients of Granular Gases
}

\author{
J. Javier Brey and David Cubero \\ Area de Física Teórica. Facultad de Física. Universidad de Sevilla. Apartado de \\ Correos 1065, 41080 Sevilla, Spain. e-mail: brey@cica.es
}

\begin{abstract}
Some transport properties of granular gases are investigated. Starting from a kinetic theory level of description, the hydrodynamic transport equations to Navier-Stokes order are presented. The equations are derived by means of the Chapman-Enskog procedure. To test the existence of a normal solution and the possibility of a hydrodynamic description, the theoretical predictions are compared with numerical simulations of the underlying kinetic equation for small deviations around the reference homogeneous state. An excellent agreement is found for all the range of dissipation in collisions considered. Similar analysis is presented for self-diffusion and Brownian motion. In the former case, also Molecular Dynamics results are shown to agree with the theoretical predictions. Quantitative and also qualitative differences with the elastic limit are discussed.
\end{abstract}

\section{Introduction}

Granular media in the so-called rapid flow regime are often described by means of continuum hydrodynamic equations [1]. The possibility of such a macroscopic description for systems with inelastic collisions is suggested by analogy with normal fluids. Dissipation in collisions is accounted for by introducing a source term in the evolution equation for the temperature. As a consequence, there is no homogeneous steady equilibrium state, but the simplest solution is given by a uniform system cooling constantly in time. Nevertheless, the justification for a hydrodynamic description, the form of the corresponding transport equations, the explicit expressions of the transport coefficients appearing in them, and the range of validity of the theory, require a detailed derivation from a more fundamental microscopic basis. As it is the case for molecular systems, the kinetic theory provides the right starting description from which the above questions can be addressed.

The simplest possibility of modeling granular flows at the particle level is as a system composed by identical smooth hard spheres or disks which collide inelastically. Moreover, the coefficient of restitution is supposed to be independent of the velocities of the colliding particles. The general formalism based on the (pseudo-)Liouville equation and also the Boltzmann and Enskog equations are easily generalized to the inelastic case [2]. In fact, several derivations of the transport equations to Navier-Stokes order for inelastic systems by applying the Chapman-Enskog method to the kinetic equations have been

T. Pöschel and S. Luding (Eds.): LNP 564, pp. 59-78, 2001

(C) Springer-Verlag Berlin Heidelberg 2001 
presented in the last decade or so [3-6]. Nevertheless, the technical difficulties following from the inelasticity in collisions has led to the introduction of approximations not required in the elastic case. These approximations restricted the validity of the resulting equations to the low dissipation or quasi-elastic limit.

Recently $[7,8]$, the above analysis is extended to arbitrary inelasticity, and the hydrodynamic fluxes and transport coefficients have been determined as functions of the coefficient of restitution. Also the cooling rate in the equation for the temperature has been analyzed to second order in the gradients, and its linear part computed explicitly. It has been found that the linear second order contributions give very small corrections to the linearized equations, so that they can be accurately neglected in linear analysis. Consequently, it is likely that the same happens with the nonlinear in the gradients part and the relevant contribution of the cooling rate to the transport equations be simply given by its zeroth order in the gradients limit.

Of course, the analogy between rapid granular flows and molecular fluids can be extended to many other transport situations. Two particularly simple cases, allowing detailed analysis are self-diffusion and Brownian motion $[9,10]$. Both processes can be considered in the low density limit, in which they are described by the Boltzmann-Lorentz equation. Their study has attracted much attention in molecular gases due to their simplicity, their close relationship with experimental situations, and also because of the possibility of a direct comparison of the theoretical predictions with computer experiments. A great deal of relevant information about dynamical processes in gases has been obtained from the analysis of diffusion data. The same is expected to happen for granular flows, especially taking into account the existence of phenomena such as density clustering, compaction, and segregation [11] that seem to be closely related to diffusion. In fact, self-diffusion in granular systems has already been the subject previous works. Macroscopic flows $[12]$ and vertically vibrated systems $[13,14]$ have been considered experimentally. Also, Molecular Dynamics simulations have been used to compute the self-diffusion coefficient in a sheared cell [15]. In this context, let us point out that knowing the explicit expression of the diffusion coefficient can be a necessary ingredient in order to determine the granular temperature in three-dimensional flows when using techniques which do not have enough time resolution to measure the temperature distribution directly.

The motion of a Brownian particle in a molecular fluid is described by the Fokker-Planck equation, that for a dilute gas can be derived from the Boltzmann-Lorentz equation in the limit of asymptotically large relative mass for the tagged particle $[9,16]$. The generalization to the inelastic case has been also considered [17]. Some interesting quantitative and also qualitative differences occur, but the point we want to stress here is that a hydrodynamic description, characterized by a diffusion equation, still holds. 
The direct simulation Monte Carlo (DSMC) method [18] provides a way for testing numerically the (analytical) theoretical predictions derived from the Boltzmann equation. Particularly interesting is the possibility of verifying the validity of a hydrodynamic description for granular systems, beyond the quasielastic limit, a point that has been a topic of interest and controversy [19-21]. Here we will report results obtained by applying the method to both the nonlinear inelastic Boltzmann equation and to the Boltzmann-Lorentz equation.

In the context of the simulation of the Boltzmann-Lorentz equation, it is worth to insist on the nature of the DSMC method as initially formulated. This method was not proposed to describe the dynamics of the particles in a low density gas, but to provide a numerical solution of an integro-differential equation, namely the nonlinear Boltzmann equation. The particles in the simulation do not correspond to real particles in the system. The number of the former can be as large as wanted and, nevertheless, one still remains in the low density limit, since it is the Boltzmann equation what is being simulated. Of course, it is possible to modify the "rules" of the simulation algorithm trying to incorporate physical effects that are not accounted for in the Boltzmann equation, but we believe this is outside the spirit of the original DSMC method. Here, we will modify the algorithm as to adjust it to the Boltzmann-Lorentz equation. This means that the distribution function of the bath, which determines the fluid "seen" by the tagged particle, is an input for the numerical simulation (as it is for the own kinetic equation). In this way, we are just numerically solving the considered kinetic equation.

The aim of this paper is to offer a short review of some recent results obtained in relation with the three above mentioned problems: Navier-Stokes transport coefficients, self-diffusion, and Brownian motion, in a granular gas. More concretely, we will focus on the possibility of a hydrodynamic description and the reasons why it is expected to be valid. Simulations of the kinetic equations will be compared with the theoretical predictions derived by assuming the existence of a Chapman-Enskog normal solution, in which the existence of the hydrodynamic level of description is inherent.

\section{Navier-Stokes Transport Coefficients}

We consider a system of smooth hard spheres $(\mathrm{d}=3)$ or disks $(\mathrm{d}=2)$ of mass $m$ and diameter $\sigma$. The particles collide inelastically and the dissipation in collisions is characterized by a constant coefficient of normal restitution $\alpha$. In the low density limit, the time evolution of the one-particle distribution function of the gas, $f(\mathbf{r}, \mathbf{v}, t)$, is assumed to be described by the Boltzmann equation $[2,6]$

$$
\left(\frac{\partial}{\partial t}+\boldsymbol{v}_{1} \cdot \boldsymbol{\nabla}\right) f\left(\boldsymbol{r}, \boldsymbol{v}_{1}, t\right)=J\left[\boldsymbol{r}, \boldsymbol{v}_{1} \mid f(t)\right],
$$


where $J$ is the (inelastic) Boltzmann collision operator,

$$
\begin{aligned}
J\left[\boldsymbol{r}, \boldsymbol{v}_{1} \mid f(t)\right]= & \sigma^{d-1} \int d \boldsymbol{v}_{2} \int d \widehat{\boldsymbol{\sigma}} \Theta(\widehat{\boldsymbol{\sigma}} \cdot \boldsymbol{g})(\widehat{\boldsymbol{\sigma}} \cdot \boldsymbol{g})\left[\alpha^{-2} f\left(\boldsymbol{r}, \boldsymbol{v}_{1}^{\prime}, t\right) f\left(\boldsymbol{r}, \boldsymbol{v}_{2}^{\prime}, t\right)\right. \\
& \left.-f\left(\boldsymbol{r}, \boldsymbol{v}_{1}, t\right) f\left(\boldsymbol{r}, \boldsymbol{v}_{2}, t\right)\right] .
\end{aligned}
$$

Here $\widehat{\boldsymbol{\sigma}}$ is a unit vector along the line joining the centers of particles 2 and 1 at contact, away from the former, $\boldsymbol{g}=\boldsymbol{v}_{1}-\boldsymbol{v}_{2}$ is the relative velocity, and $\Theta$ is the Heaviside step function. The velocities $\boldsymbol{v}_{1}^{\prime}, \boldsymbol{v}_{2}^{\prime}$ are the precollisional velocities leading after collision to velocities $\boldsymbol{v}_{1}, \boldsymbol{v}_{2}$. They are given by

$$
\boldsymbol{v}_{1}^{\prime}=\boldsymbol{v}_{1}-\frac{1+\alpha}{2 \alpha}(\widehat{\boldsymbol{\sigma}} \cdot \boldsymbol{g}) \widehat{\boldsymbol{\sigma}}, \text { and } \boldsymbol{v}_{2}^{\prime}=\boldsymbol{v}_{2}+\frac{1+\alpha}{2 \alpha}(\widehat{\boldsymbol{\sigma}} \cdot \boldsymbol{g}) \widehat{\boldsymbol{\sigma}}
$$

The macroscopic balance equations are obtained from Eq. (1) by multiplying with $1, m \boldsymbol{v}_{1}$, and $m v_{1}^{2}$ and integrating over $\boldsymbol{v}_{1}$,

$$
\begin{aligned}
\partial_{t} n+\nabla \cdot(n \boldsymbol{u}) & =0, \\
\partial_{t} \boldsymbol{u}+\boldsymbol{u} \cdot \boldsymbol{\nabla} \boldsymbol{u}+(n m)^{-1} \boldsymbol{\nabla} \cdot \mathrm{P} & =0, \\
\partial_{t} T+\mathbf{u} \cdot \boldsymbol{\nabla} T+2\left(d n k_{B}\right)^{-1}(\mathrm{P}: \boldsymbol{\nabla} \boldsymbol{u}+\boldsymbol{\nabla} \cdot \boldsymbol{q})+T \zeta & =0 .
\end{aligned}
$$

The local particle number density $n$, flow velocity $\boldsymbol{u}$, and temperature $T$ are defined in the usual way,

$$
\begin{aligned}
n(\boldsymbol{r}, t) & =\int d \boldsymbol{v} f(\boldsymbol{r}, \boldsymbol{v}, t), \\
n(\boldsymbol{r}, t) \boldsymbol{u}(\boldsymbol{r}, t) & =\int d \boldsymbol{v} \boldsymbol{v} f(\boldsymbol{r}, \boldsymbol{v}, t), \\
\frac{d}{2} n(\boldsymbol{r}, t) k_{B} T(\boldsymbol{r}, t) & =\int d \boldsymbol{v} \frac{m V^{2}}{2} f(\boldsymbol{r}, \boldsymbol{v}, t),
\end{aligned}
$$

where $k_{B}$ is the Boltzmann constant and $\boldsymbol{V}(\boldsymbol{r}, t)=\boldsymbol{v}-\boldsymbol{u}(\boldsymbol{r}, t)$. In Eqs. (4a)(4c) the pressure tensor $\mathrm{P}$, and the heat flux $\boldsymbol{q}$ are given by

$$
\begin{aligned}
\mathrm{P}(\boldsymbol{r}, t) & =\int d \boldsymbol{v} m \boldsymbol{V} \boldsymbol{V} f(\boldsymbol{r}, \boldsymbol{v}, t), \\
\boldsymbol{q}(\boldsymbol{r}, t) & =\int d \boldsymbol{v} \frac{m V^{2}}{2} \boldsymbol{V} f(\boldsymbol{r}, \boldsymbol{v}, t) .
\end{aligned}
$$

Finally, the cooling rate $\zeta$ in the equation for the temperature (4c) takes into account the energy dissipation in collisions, and it is a nonlinear functional of the distribution function,

$$
\zeta(\boldsymbol{r}, t)=\frac{\left(1-\alpha^{2}\right) m \pi^{\frac{d-1}{2}} \sigma^{d-1}}{4 d \Gamma\left(\frac{d+3}{2}\right) n k_{B} T} \int d \boldsymbol{v}_{1} \int d \boldsymbol{v}_{2} g^{3} f\left(\boldsymbol{r}, \boldsymbol{v}_{1}, t\right) f\left(\boldsymbol{r}, \boldsymbol{v}_{2}, t\right)
$$

Macroscopic balance equations similar to Eqs. (4a)-(4c) have been derived many times in the literature [3-5]. Of course, they only become closed hydrodynamic equations once the pressure tensor, the heat flux, and the cooling 
rate are expressed as functionals of the macroscopic fields. In principle this can be achieved by means of a Chapman-Enskog expansion, in the same spirit as for elastic molecular gases. Nevertheless, the complexity introduced by the energy dissipation in collisions has led to the introduction of some additional approximations, restricting the validity of the results to the small inelasticity limit. Only very recently explicit expressions for the fluxes to first order in the gradients as explicit functions of the coefficient of restitution have been obtained [7]. The expressions read

$$
\begin{gathered}
P_{i j}=n k_{B} T \delta_{i j}-\eta\left(\nabla_{i} u_{j}+\nabla_{j} u_{i}-\frac{2}{d} \delta_{i j} \nabla \cdot \boldsymbol{u}\right), \\
\boldsymbol{q}=-\kappa \nabla T-\mu \nabla n
\end{gathered}
$$

where $\eta$ is the shear viscosity, $\kappa$ the thermal conductivity, and $\mu$ a new transport coefficient, which has no analogue in elastic gases, coupling density gradient and heat flux. These transport coefficients are given by

$$
\begin{gathered}
\eta^{*}(\alpha) \equiv \frac{\eta(\alpha)}{\eta_{0}}=\left[\nu_{1}^{*}(\alpha)-\frac{\zeta^{*}(\alpha)}{2}\right]^{-1} \\
\kappa^{*}(\alpha) \equiv \frac{\kappa(\alpha)}{\kappa_{0}}=\left[\nu_{2}^{*}(\alpha)-\frac{2 d}{d-1} \zeta^{*}(\alpha)\right]^{-1}\left[1+c^{*}(\alpha)\right] \\
\mu^{*}(\alpha) \equiv \frac{n}{T \kappa_{0}} \mu(\alpha) \\
=2 \zeta^{*}(\alpha)\left[\kappa^{*}(\alpha)+\frac{(d-1) c^{*}(\alpha)}{2 d \zeta^{*}(\alpha)}\right]\left[\frac{2(d-1)}{d} \nu_{2}^{*}(\alpha)-3 \zeta^{*}(\alpha)\right]^{-1}
\end{gathered}
$$

In the above expressions

$$
\begin{aligned}
& \eta_{0}=\frac{2+d}{8} \Gamma(d / 2) \pi^{-\frac{d-1}{2}}\left(m k_{B} T\right)^{1 / 2} \sigma^{-(d-1)}, \text { and } \\
& \kappa_{0}=\frac{d(d+2)^{2}}{16(d-1)} \Gamma(d / 2) \pi^{-\frac{d-1}{2}} k_{B}\left(\frac{k_{B} T}{m}\right)^{1 / 2} \sigma^{-(d-1)}
\end{aligned}
$$

are the values in a molecular gas of the shear viscosity and thermal conductivity, respectively. The dimensionless functions of the coefficient of restitution introduced in Eqs. (11)-(13) have the expressions

$$
\begin{gathered}
\zeta^{*}(\alpha)=\frac{2+d}{4 d}\left(1-\alpha^{2}\right)\left[1+\frac{3}{32} c^{*}(\alpha)\right], \\
\nu_{1}^{*}(\alpha)=\frac{(3-3 \alpha+2 d)(1+\alpha)}{4 d}\left[1-\frac{1}{64} c^{*}(\alpha)\right],
\end{gathered}
$$




$$
\begin{gathered}
\nu_{2}^{*}=\frac{1+\alpha}{d-1}\left[\frac{d-1}{2}+\frac{3(d+8)(1-\alpha)}{16}+\frac{4+5 d-3(4-d) \alpha}{1024} c^{*}(\alpha)\right], \\
c^{*}(\alpha)=\frac{32(1-\alpha)\left(1-2 \alpha^{2}\right)}{9+24 d+(8 d-41) \alpha+30 \alpha^{2}(1-\alpha)} .
\end{gathered}
$$

The energy sink term has the form $\zeta=\zeta^{(0)}+\zeta^{(2)}$, where $\zeta^{(0)}$ denotes the zeroth order in the gradients contribution,

$$
\zeta^{(0)}=\zeta^{*} \frac{n k_{B} T}{\eta_{0}}
$$

while $\zeta^{(2)}$ is of second order in the gradients. Only its linear in the gradients part, $\zeta_{l}^{(2)}$, must be considered for the linear analysis we will present in the following. It has the form

$$
\zeta_{l}^{(2)}=\zeta_{1} \nabla^{2} T+\zeta_{2} \nabla^{2} n
$$

The expressions for the transport coefficient $\zeta_{1}$ and $\zeta_{2}$ are quite involved and not particularly relevant for the purposes here, since they give contributions to the transport equations that can be accurately neglected [7].

A point to be noted is that the above expressions for the transport coefficients have been obtained in the so-called first Sonine approximation, in which the distribution function of the gas is expanded in Sonine polynomials and only the first corrections to the Gaussian giving contributions to the several fluxes are retained. This approximation is also usual in molecular gases where it has been proved to be quite accurate. It is expected to hold also for inelastic systems since the reference state is Gaussian with very good approximation $[6,7,22]$. Let us also point out that the above analysis has been very recently extended to the revised Enskog kinetic theory for hard spheres $[2,23]$, providing then a macroscopic description at higher densities [8].

The granular hydrodynamic equations admit a solution describing the homogeneous cooling state (HCS), characterized by uniform fields and a time dependent temperature $T_{H}(t)$ obeying the equation

$$
\frac{\partial}{\partial t} T_{H}(t)=-\zeta^{(0)}(t) T_{H}(t)
$$

We want to investigate the validity of the hydrodynamic description for states close to the HCS. Then we define deviations by

$$
n(\boldsymbol{r}, t)=n+\delta n(\boldsymbol{r}, t), \boldsymbol{u}(\boldsymbol{r}, t)=\delta \boldsymbol{u}(\boldsymbol{r}, t), T(\boldsymbol{r}, t)=T_{H}(t)+\delta T(\boldsymbol{r}, t),
$$

where $n$ is the average density of the system. Linearization of Eqs. (4a)-(4c) about the HCS leads to partial differential equations with time dependent coefficients which are not suitable for a direct linear stability analysis. This 
is a consequence of the time dependence of the reference state, the HCS, and can be eliminated through a change of time and space variables, and a scaling of the hydrodynamic fields. We define

$$
\boldsymbol{l}=\frac{\nu_{H}(t)}{2} v_{H}^{-1}(t) \boldsymbol{r}, \quad \tau=\frac{1}{2} \int_{0}^{t} d t^{\prime} \nu_{H}\left(t^{\prime}\right),
$$

where $\nu_{H}(t)=n k_{B} T_{H} / \eta_{0}\left(T_{H}\right)$ is a characteristic frequency, and $v_{H}(t)=$ $\left(k_{B} T_{H} / m\right)^{1 / 2}$ is the thermal velocity. Note that the length scale transformation is time independent. The scaled fields are

$$
\rho(\boldsymbol{l}, \tau)=\frac{\delta n(\boldsymbol{l}, \tau)}{n}, \quad \boldsymbol{\omega}(\boldsymbol{l}, \tau)=\frac{\delta \boldsymbol{u}(\boldsymbol{l}, \tau)}{v_{H}(\tau)}, \quad \theta(\boldsymbol{l}, \tau)=\frac{\delta T(\boldsymbol{l}, \tau)}{T_{H}(\tau)} .
$$

In the remainder of this Section, we will restrict ourselves to the particular case of hard spheres for the sake of simplicity, i. e. we take $d=3$. When the new variables and fields are used, the linearized hydrodynamic equations become

$$
\begin{gathered}
\partial_{\tau} \rho_{\boldsymbol{k}}+i k w_{\boldsymbol{k} \|}=0 \\
\left(\partial_{\tau}-\zeta^{*}+\frac{2}{3} \eta^{*} k^{2}\right) w_{\boldsymbol{k} \|}+i k \theta_{\boldsymbol{k}}+i k \rho_{\boldsymbol{k}}=0 \\
\left(\partial_{\tau}-\zeta^{*}+\frac{1}{2} \eta^{*} k^{2}\right) \boldsymbol{w}_{\boldsymbol{k} \perp}=0 \\
\left(\partial_{\tau}+\zeta^{*}+\frac{5}{4} \kappa^{*} k^{2}\right) \theta_{\boldsymbol{k}}+\left(2 \zeta^{*}+\frac{5}{4} \mu^{*} k^{2}\right) \rho_{\boldsymbol{k}}+\frac{2}{3} i k w_{\boldsymbol{k} \|}=0 .
\end{gathered}
$$

We have introduced the Fourier transformed of the hydrodynamic fields defined by

$$
\rho_{\boldsymbol{k}}(\tau)=\int d \boldsymbol{l} e^{-i \boldsymbol{k} \cdot \boldsymbol{l}} \rho(\boldsymbol{l}, \tau)
$$

and so on. Besides, $w_{\boldsymbol{k} \|}$ and $\boldsymbol{w}_{\boldsymbol{k} \perp}$ are the longitudinal and transversal components of the velocity field relative to the wave vector $\boldsymbol{k}$, respectively. From Eq. (28), the time evolution of the transversal components of the velocity field is directly obtained

$$
\boldsymbol{w}_{\boldsymbol{k} \perp}(\tau)=\boldsymbol{w}_{\boldsymbol{k} \perp}(0) e^{s_{\perp} \tau}
$$

where the eigenvalue $s_{\perp}$ associated to these "shear modes" is

$$
s_{\perp}=\zeta^{*}-\frac{1}{2} \eta^{*} k^{2} .
$$

Taking into account the definitions in Eq. (25) and that from Eq. (22) it follows that $T_{H}(\tau)=T_{H}(0) \exp \left(-2 \zeta^{*} \tau\right)$, Eq. (31) leads to

$$
\boldsymbol{u}_{\boldsymbol{k} \perp}(\tau)=\boldsymbol{u}_{\boldsymbol{k} \perp}(0) e^{-\frac{\eta^{*} k^{2} \tau}{2}},
$$


i.e. perturbations of the transversal component of the velocity always decay in time. Let us remark that an exponential behavior in the reduced variable $\tau$ translates into an algebraic decay in the actual time $t$. It is easily seen from Eqs. (22) and (24) that

$$
e^{\tau s}=\left(1+\frac{t}{t_{0}}\right)^{s / \zeta^{*}}
$$

with $t_{0}^{-1}=\zeta^{*} \nu_{H}(0)$.

The above hydrodynamic description has been derived from the (inelastic) Boltzmann equation by assuming that the generalization of the ChapmanEnskog method can be used in order to obtain a normal solution to the kinetic equation. This requires a clear separation between the time scale governing the kinetic excitations of the system and the much larger time scale on which the macroscopic fields change in time. This separation is well established in the case of molecular gases, but the situation is more complicated when the collisions are not elastic. The time evolution of the hydrodynamic fields is not determined only by their spatial gradients, but there is another time scale for the temperature, set up by the inelasticity of the system through the homogeneous cooling rate $\zeta^{(0)}$. In this sense, it could be said that there are two hydrodynamic time scales in rapid granular flows: one associated to the macroscopic gradients and another one following directly from dissipation in collisions. Nevertheless, the point is not whether these scales are or are not separated one from the other, but whether they are both much larger than the one associated to the (microscopic) kinetic excitations.

A direct check of the accuracy of the hydrodynamic equations is provided by the comparison of solutions of them with solutions to the Boltzmann equation in which no hydrodynamic concepts have been introduced externally. Numerical solutions of the Boltzmann equation can be constructed by means of the direct simulation Monte Carlo (DSMC) method [18]. The general idea of the method is to generate a Markov process which mimics the dynamical processes described by the kinetic equation. Inelasticity in collisions is incorporated just by changing the expressions of the postcollisional velocities as compared with the elastic case. Since the details of the method have been discussed many times in the literature and can be found in Ref. [18], they will not be given here.

The kind of initial conditions we have considered corresponds to small amplitude perturbations about the HCS reached by a freely evolving granular gas, so that the linearized hydrodynamic equations (26)-(29) are expected to hold. The easiest macroscopic perturbation one can think of consists in an initial harmonic perturbation of the transversal component of the velocity field given by

$$
u_{y}(x, 0)=u_{0} \sin \left(q_{0} x\right)
$$

where $u_{0}=0.1 \sqrt{2} v_{H}(0)$ and $q_{0}=2 \pi / L, L$ being the size of the system in the $x$-direction. Along this direction, periodic boundary conditions are applied in 
the simulation of the Boltzmann equation. According to Eq. (31), the time evolution of the perturbation follows the law:

$$
u_{y}(x, \tau)=u_{\tau} \sin \left(q_{0} x\right), \quad u_{\tau}=u_{0} e^{-\eta^{*} k_{0}^{2} \tau / 2} .
$$

Here $k_{0}$ is the dimensionless reduced wavenumber corresponding to $q_{0}$, i.e. $k_{0}=2 \nu_{H}^{-1} v_{H} q_{0}$. The simulation results show the qualitative behavior described above, i.e. the transversal component of the velocity flow $u_{y}$ has a profile along the $x$-direction that can be accurately fitted by a sine function with an amplitude decreasing exponentially with the scaled time $\tau$ [24]. This provides a numerical value for the reduced shear viscosity $\eta^{*}$ in Eq. (36). The results for several values of the restitution coefficient $\alpha$ are compared with the theoretical prediction given by Eq. (11) in Fig. 1. It is seen that there is a fairly good agreement over the wide range of $\alpha$ values considered, along which the variation of the shear viscosity coefficient is of the order of $20 \%$. Close inspection of Fig. 1 indicates a small but systematic discrepancy

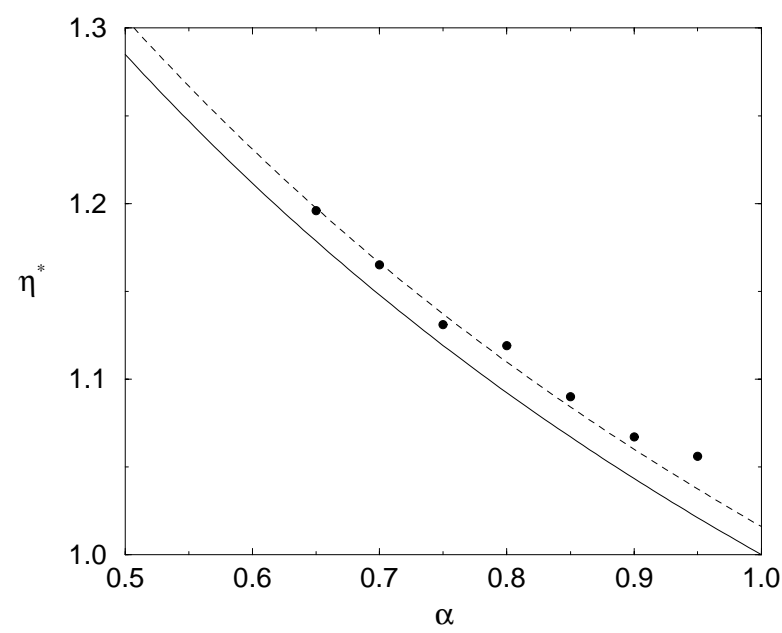

Fig. 1. Reduced shear viscosity $\eta^{*}$ as a function of the coefficient of restitution $\alpha$. The solid line is the theoretical prediction obtained by the Chapman-Enskog method in the first Sonine approximation and the points are from the DSMC method. The dashed line has been obtained by using a correction factor derived in the elastic limit $\alpha=1$

between theory and simulation. Results from the latter always lay above the theoretical curve. This seems to be a consequence of the use of the first Sonine approximation upon deriving the hydrodynamic transport coefficients. In the elastic limit, a correction factor of the order of 1.016 to the first Sonine expression for the shear viscosity $\eta_{0}$ has been obtained [25]. The dashed curve in Fig. 1 has been constructed by using this factor for all $\alpha$. 
Since the time evolution of the density also depends on the values of other hydrodynamic fields, a more complicated initial perturbation is

$$
\rho(x, 0)=\rho_{0} \sin \left(q_{0} x\right) .
$$

Now the solution of the linearized hydrodynamic equations can not be written down in a simple way. The time evolution of the amplitudes of the reduced density, temperature, and longitudinal component of the flow field is given by the linear combination of three exponentials, in the reduced time scale. The values of the relaxation times in the exponents are the roots of a cubic equation defining the dispersion relations [7].

In Fig. 2 we present the time evolution of the Fourier amplitudes of the hydrodynamic fields after introducing at $t=0$ a perturbation given by Eq. (37) with $\rho_{0}=0.1$ (Note a factor of 2 of difference between the amplitude of the sine function and the corresponding Fourier component). The longitudinal component of the velocity field has not been plotted since it remains very small, below the noise level. The coefficient of restitution in the simulations shown in the figure is $\alpha=0.7$. A good agreement is observed between the numerical solution of the Boltzmann equation and the predictions from the linearized hydrodynamic equations. Similar results have been found for several values of $\alpha$ in the interval $0.7 \leq \alpha \leq 0.95$ [24].

The above results confirm the validity of the hydrodynamic picture, as derived by means of the Chapman-Enskog procedure, to describe the time evolution of the macroscopic fields of a dilute granular gas whose time evolution is governed by the Boltzmann equation, at least for states close the HCS. Let us point out that results obtained for the time evolution of a linear density perturbation by Molecular Dynamic simulations of inelastic hard disks [26] are consistent with those presented above. For large times, one of the eigenmodes dominates the evolution of the hydrodynamic fields in the linear approximation. This is why the curves in the figure become straight lines. The expression for the corresponding eigenvalue involves all the transport coefficients [7].

\section{Self-Diffusion}

Let us now consider that some of the particles in the gas are labeled, but are otherwise identical to the others. The tagged particles will be described by the one particle distribution function $f_{s}(\boldsymbol{r}, \boldsymbol{v}, t)$. It will be assumed that the gas as a whole is in the HCS, and its distribution function will be denoted by $f_{H}(\boldsymbol{v}, t)$. Then, $f_{s}$ obeys the Boltzmann-Lorentz equation,

$$
\begin{gathered}
\left(\partial_{t}+\boldsymbol{v}_{1} \cdot \boldsymbol{\nabla}\right) f_{s}\left(\boldsymbol{r}, \boldsymbol{v}_{1}, t\right)=\sigma^{d-1} \int d \boldsymbol{v}_{2} \int d \widehat{\boldsymbol{\sigma}} \Theta(\widehat{\boldsymbol{\sigma}} \cdot \boldsymbol{g})(\widehat{\boldsymbol{\sigma}} \cdot \boldsymbol{g}) \\
\times\left[\alpha^{-2} f_{s}\left(\boldsymbol{r}, \boldsymbol{v}_{1}^{\prime}, t\right) f_{H}\left(\boldsymbol{v}_{2}^{\prime}, t\right)-f_{s}\left(\boldsymbol{r}, \boldsymbol{v}_{1}, t\right) f_{H}\left(\boldsymbol{v}_{2}, t\right)\right]
\end{gathered}
$$




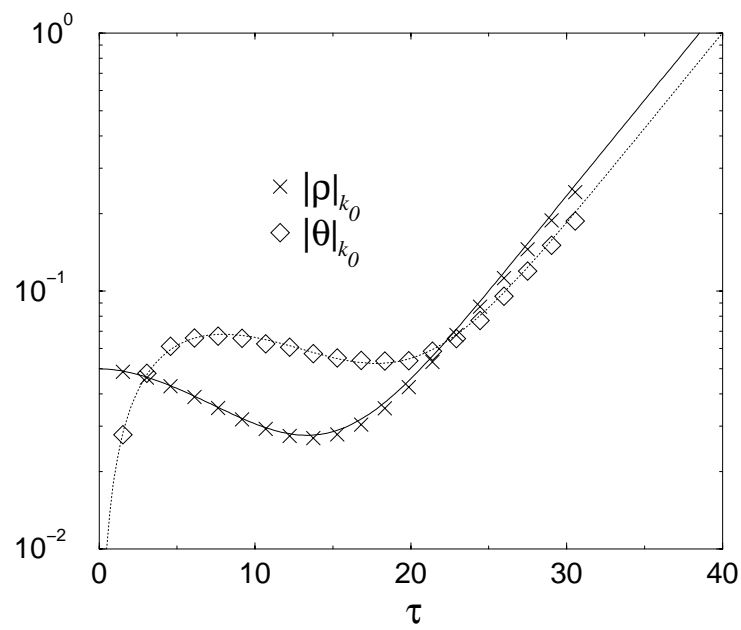

Fig. 2. Time evolution of Fourier components of the scaled density $\rho$ and temperature $\theta$, following an harmonic perturbation of the density. The symbols are from DSMC method and the lines from the linear hydrodynamic equations. All quantities are measured in the dimensionless units defined in the main text

Here we are using the same notation as in Eqs. (1)-(2).

The Boltzmann-Lorentz equation is based on the same hypothesis as the nonlinear Boltzmann equation and is restricted to the low density limit. Higher densities can be addressed by means of the (inelastic) Enskog equation [2] or, in the particular case of self-diffusion, the Enskog-Lorentz equation. For homogeneous systems, the only difference between the Enskog and Boltzmann description of self-diffusion is in the presence of the equilibrium pair correlation function of the system at distance $\sigma, g_{e}(n)$, as a factor in front of the collision integral [10]. Therefore, one can translate results obtained for the Boltzmann-Lorentz equation into results for Enskog-Lorentz equation by simply substituting $\sigma^{d-1}$ by $g_{e}(n) \sigma^{d-1}$.

The density of tagged particles

$$
n_{s}(\boldsymbol{r}, t)=\int d \boldsymbol{v} f_{s}(\boldsymbol{r}, \boldsymbol{v}, t)
$$

obeys the conservation law

$$
\partial_{t} n_{s}(\boldsymbol{r}, t)=-\boldsymbol{\nabla} \cdot \boldsymbol{J}_{s}(\boldsymbol{r}, t),
$$

where $\boldsymbol{J}_{s}$ is the flux of tagged particles

$$
\boldsymbol{J}_{s}(\boldsymbol{r}, t)=\int d \boldsymbol{v} \boldsymbol{v} f_{s}(\boldsymbol{r}, \boldsymbol{v}, t) .
$$

By using the Chapman-Enskog method it is possible to obtain a normal solution to the Enskog-Lorentz equation valid to first order in the gradient 
of the density of tagged particles [27]. Then the flux of tagged particles can be computed. The final result is a diffusion equation of the form

$$
\partial_{t} n_{s}(\boldsymbol{r}, t)=-D(t) \nabla^{2} n_{s}(\boldsymbol{r}, t)
$$

with a time dependent self-diffusion coefficient $D(t)$ given by

$$
D(t)=D_{E}\left(T_{H}\right) D^{*}(\alpha)
$$

where $D_{E}$ is the Enskog self-diffusion coefficient in an elastic system,

and

$$
D_{E}=\frac{d \Gamma(d / 2)}{4 \pi^{\frac{d-1}{2}} n g_{e}(n) \sigma^{d-1}}\left(\frac{k_{B} T_{H}}{m}\right)^{1 / 2}
$$

$$
D^{*}(\alpha)=\frac{4}{(1+\alpha)^{2}-\frac{c^{*}}{32}\left(4+\alpha-3 \alpha^{2}\right)} .
$$

Of course, the Boltzmann limit is obtained by taking $g_{e}(n)=1$. The selfdiffusion coefficient in Eq. (42) depend on time through the granular temperature $T_{H}(t)$. This time dependence can be eliminated by using again the time and space scales defined by Eq. (24). To get a simpler result, instead of the frequency $\nu_{H}$ given below Eq. (24) we use here

$$
\nu_{0}=\frac{2 k_{B} T}{m D_{E}\left(T_{H}\right)} .
$$

In the reduced variables Eq. (42) reads

$$
\partial_{\tau} \rho_{s}(\boldsymbol{l}, \tau)=D^{*}(\alpha) \nabla_{l}^{2} \rho_{s}(\boldsymbol{l}, \tau)
$$

where $\rho_{s}=n_{s} / n$. Now we have a diffusion equation with a constant diffusion coefficient. It follows that the mean square displacement of the scaled position $\boldsymbol{l}$ of the tagged particles is given by

$$
\left\langle(\Delta \boldsymbol{l})^{2} ; \tau\right\rangle=2 d D^{*}(\alpha) \tau
$$

To test the applicability of the Chapman-Enskog procedure to the inelastic self-diffusion problem, the DSMC method has been applied to the BoltzmannLorentz equation for hard spheres. As indicated in Eq. (38), the distribution function of the complete gas is required for input. Consistently with the theory we have developed, the distribution is taken to be the homogeneous cooling solution to the Boltzmann equation. More concretely, we have used the expression obtained in the first Sonine approximation $[6,28]$. As a consequence, the problem of the clustering instability of the HCS for large wavelengths and strong dissipation $[22,29]$ can not be addressed in these simulations.

Two different procedures have been used to measure numerically the selfdiffusion coefficient. In the first one, the scaled mean square displacement 
$\left\langle(\Delta l)^{2}\right\rangle$ was measured as a function of the reduced time $\tau$. After a short transient time, a linear behavior was found, in agreement with Eq. (48). The slope of the straight line fitting the numerical data provides the value for $D^{*}$. In the second method, an initial density of tagged particles perturbation was introduced,

$$
n_{s}(x, 0)=n_{0}\left[1+\sin \left(q_{0} x\right)\right],
$$

where, again, $q_{0}=2 \pi / L$ and periodic boundary conditions along the $x$ direction are employed. Notice that, contrary to the linear analysis of the Navier-Stokes equations presented in the previous Section, now we are not restricted to the small perturbation limit, and the precise value of $n_{0}$ is not relevant here. The diffusion equation predicts that

$$
n_{s}(x, \tau)=n_{0}\left[1+e^{-s_{D} \tau} \sin \left(q_{0} x\right)\right],
$$

with $s_{D}=D^{*} k_{0}^{2}$. Again $k_{0}$ denotes the dimensionless wave number corresponding to $q_{0}$. Then, by following the time decay of the amplitude of the sine perturbation, a numerical value for $D^{*}$ follows. An example of the time evolution of the density profile is given in Fig. 3, where it is plotted at three different times. The coefficient of normal restitution is $\alpha=0.95$. It is seen that the perturbation it is accurately described by a sine function with a time dependent amplitude.

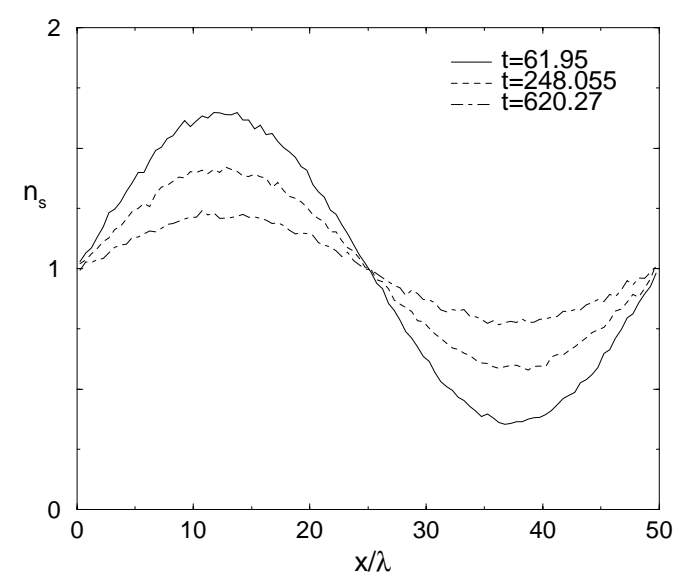

Fig. 3. Density profile along the $x$-direction at three different times following a sine initial perturbation with a wavelength determined by the size of the system. The density is normalized with the average density, and length is measured in units of the mean free path $\lambda=\left(\sqrt{2} n_{0} \pi \sigma^{2}\right)^{-1}$. The indicated times have been scaled as indicated in Eq. (24), but with the frequency $\nu_{0}$

Fig. 4 shows the comparison of the two simulation experiments we have described with the theoretical prediction given by Eq. (45) for several values 
of the coefficient of restitution. A fairly good agreement is observed for all the range of values of $\alpha$ considered, namely $0.6 \leq \alpha \leq 1$.

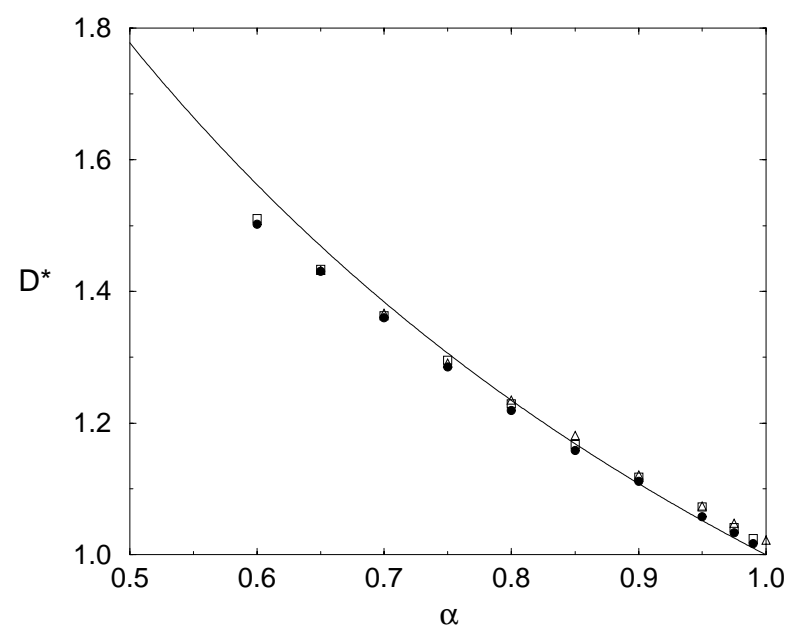

Fig. 4. Reduced self-diffusion coefficient $D^{*}$ as a function of the coefficient of normal restitution $\alpha$. The solid line is the theoretical prediction derived from the Boltzmann-Lorentz equation by using the Chapman-Enskog procedure, the circles and squares are numerical values obtained from the direct Monte Carlo simulation of the kinetic equation by using the mean square displacement and a sine perturbation in density, respectively, and the triangles are from Molecular Dynamics simulations

The DSMC method provides a way for checking the validity of theoretical results derived from the Boltzmann (or Enskog) kinetic equation. In particular, we have used it above to verify the existence of normal or hydrodynamic solutions. A different and also fundamental question is whether the own kinetic equation gives an accurate description of the time evolution of the system of particles it is expected to represent. In order to address this second issue, we have carried out Molecular Dynamics simulations of a system of 6400 inelastic hard disks, with a number density $n \sigma^{2}=6.25 \cdot 10^{-4}$, that is equivalent to a solid fraction of $5 \cdot 10^{-4}$. For this density, the equilibrium pair distribution at contact is $g_{e} \simeq 1.0008$, so that we are clearly in the low density region. The quantity we computed was the mean square displacement or, more precisely, $m_{D}=\left(4 D_{E}\right)^{-1} \partial_{t}\left\langle(\Delta \boldsymbol{r})^{2}\right\rangle$. After a few collisions per particle, this quantity reached a time independent plateau, that according to Eq. (42) should correspond to the diffusion regime and give the value of the reduced self-diffusion coefficient. The values for $D^{*}$ obtained in this way have been also included in Fig. 4 . The agreement with the theoretical prediction is again very good. Quite interestingly, the Molecular Dynamics data coincide on the scale of the figure with the numerical solution of the 
Boltzmann-Lorentz equation obtained by the DSMC method. This strongly suggests that the small discrepancy between the Chapman-Enskog solution and the Molecular Dynamics results is due to the introduction of approximations when carrying out the former and, in particular, to the use of the first Sonine approximation. The situation seems to be similar to the one found in the study of the shear viscosity coefficient (see Fig. 1 and the discussion about it).

Two points deserve some additional comments before closing this Section. Firstly, in the Molecular Dynamics simulations there is no control on the state of the gas system, and it can develop cluster instabilities under the appropriate conditions. Therefore, the fact that self-diffusion behavior was observed, means that the results obtained by applying the Chapman-Enskog procedure to the Boltzmann-Lorentz equation are relevant, in the sense that a system of inelastic hard particles presents time and space windows inside which hydrodynamics provides an accurate description of the internal fluxes of particles. On the other hand, it is true than when the system becomes very dissipative, instabilities develops very soon in large systems and the "hydrodynamic window" may become very narrow. For this reason, the smallest value of the restitution coefficient for which we report a value of the diffusion coefficient from Molecular Dynamics is $\alpha=0.7$. Note that for this dissipation the self-diffusion coefficient has already increased more than $30 \%$ above the elastic limit value.

The second comment refers to the consideration of hard spheres in the DSMC method, while hard disks were used in Molecular Dynamics for efficiency reasons. It may appear as surprising that the results agree for different dimension of the system. However, it is seen in Eq. (45) that the only dependence of $D^{*}$ on $d$ occurs through $c^{*}$, and the term containing it is negligible as compared with $(1+\alpha)^{2}$.

\section{Brownian Motion}

Now let us consider a tagged particle of mass $m$ immersed in a low density gas of particles with mass $m_{g}$, i.e. the tagged particle is not mechanically equivalent to the gas particles. We will assume that all particles are hard spheres or disks colliding inelastically. The coefficient of normal restitution for the gas particle collisions will be denoted by $\alpha_{g}$ while for collisions of the tagged particle (with the gas particles) $\alpha$ will be used. As in our study of self-diffusion in the previous section, the gas will be supposed to be in the HCS.

The probability density for the tagged particle, $f_{s}(\boldsymbol{r}, \boldsymbol{v}, t)$, will obey the Boltzmann-Lorentz equation (38). In this Section the case of a very massive tagged particle will be studied. In the limit $\Delta \equiv m_{g} / m \rightarrow 0$, the equation reduces to leading order to a Fokker-Planck equation [17],

$$
\left(\partial_{t}+\boldsymbol{v} \cdot \nabla\right) f_{s}(\boldsymbol{r}, \boldsymbol{v}, t)=\mathcal{L}\left[T_{H}(t)\right] f_{s}(\boldsymbol{r}, \boldsymbol{v}, t),
$$




$$
\mathcal{L}\left(T_{H}\right)=\gamma_{e}\left(T_{H}\right) a(\alpha) \frac{\partial}{\partial \boldsymbol{v}} \cdot\left[\boldsymbol{v}+\frac{k_{B} T_{H}}{m} a(\alpha) \frac{\partial}{\partial \boldsymbol{v}}\right],
$$

where $\gamma_{e}$ is the same friction coefficients as for elastic systems, except as a function of the time dependent temperature of the gas $T_{H}(t)$,

$$
\gamma_{e}\left(T_{H}\right)=\frac{4 \pi^{\frac{d-1}{2}} \sigma_{0}^{d-1} n_{g} \Delta^{1 / 2}}{d \Gamma\left(\frac{d}{2}\right)}\left(\frac{2 k_{B} T_{H}}{m}\right)^{1 / 2} .
$$

Here $n_{g}$ is the density of the gas, $\sigma_{0}=\left(\sigma+\sigma_{g}\right) / 2$, and $a=(1+\alpha) / 2$. This latter quantity contains all the influence of the inelasticity of collision between the Brownian particle and the fluid particles. Its presence inside the square brackets in Eq. (52) implies that the usual fluctuation-dissipation for elastic particles is modified. An important and nontrivial feature is that the derivation of the above Fokker-Planck equation requires, in addition to the above-mentioned limit $\Delta \rightarrow 0$, that also $\alpha_{g} \rightarrow 1$, in such a way that

$$
\epsilon_{0} \equiv \frac{\zeta^{(0)}(t)}{2 a \gamma_{e}(t)} \rightarrow \text { constant }<1 \text {. }
$$

Again $\zeta^{(0)}(t)$ is the cooling rate for the HCS, given in Eq. (20). Therefore, the validity of the Fokker-Planck equation, as derived from the inelastic Boltzmann-Lorentz equation is restricted to the limit of weak dissipation in the gas, although there is no limitation on the inelasticity of collisions of the tagged particle.

It is convenient to define a temperature $T(t)$ of the Brownian particle from its velocity fluctuations by

$$
\frac{d}{2} k_{B} T=\int d \boldsymbol{r} \int d \boldsymbol{v} \frac{1}{2} m(\boldsymbol{v}-\boldsymbol{u})^{2} f_{s}
$$

where $\boldsymbol{u}$ is the spatial average of the macroscopic velocity field of the particle,

$$
\boldsymbol{u}(t)=\int d \boldsymbol{r} \int d \boldsymbol{v} \boldsymbol{v} f_{s} .
$$

Equation (51) has the following two properties [17]:

1. In the long time limit the temperature of the tagged particle approaches the same cooling rate as the surrounding gas, but both temperatures remain different and their ratio approaches a constant,

$$
\lim _{t \rightarrow \infty} \frac{T(t)}{T_{H}(t)}=\frac{a(\alpha)}{1-\epsilon_{0}} .
$$

Then, the asymptotic temperature of the tagged particles can be larger or smaller than that of the gas depending on whether it is $(1-\alpha) / 2<\epsilon_{0}<1$ or $0<\epsilon_{0}<(1-\alpha) / 2$. 
2. The long time limit of the probability distribution of the Brownian particle is Gaussian, even though the distribution of the surrounding gas is not,

$$
f_{s}(\boldsymbol{r}, \boldsymbol{v}, t) \rightarrow f_{s, H}(\boldsymbol{v}, t)=\Omega^{-1} \frac{\tilde{v}_{0}^{-d}(t)}{\pi^{d / 2}} e^{-\frac{v^{2}}{\tilde{v}_{0}^{2}(t)}}
$$

where $\Omega$ is the volume of the system and $\tilde{v}_{0}(t)=\left[2 k_{B} T(t) / m\right]^{1 / 2}$.

Let us write the Fokker-Planck equation in Fourier space,

$$
\left(\partial_{t}+i \boldsymbol{q} \cdot \boldsymbol{v}\right) f_{s}(\boldsymbol{q}, \boldsymbol{v}, t)=\mathcal{L}\left[T_{H}(t)\right] f_{s}(\boldsymbol{q}, \boldsymbol{v}, t),
$$

where

$$
f_{s}(\boldsymbol{q}, \boldsymbol{v}, t)=\int d \boldsymbol{r} e^{-i \boldsymbol{q} \cdot \boldsymbol{r}} f_{s}(\boldsymbol{r}, \boldsymbol{v}, t) .
$$

The eigenvalues of the operator $\mathcal{L}-i \boldsymbol{q} \cdot \boldsymbol{v}$ have the form $[9,10,30]$

$$
\lambda_{n}=-a(\alpha) \gamma_{e}\left[T_{H}(t)\right] \sum_{i=1}^{d} n_{i}-D_{e}\left[T_{H}(t)\right] q^{2},
$$

$n_{i}=0,1,2,3, \cdots$. The coefficient $D_{e}$ is the same as the elastic diffusion coefficient but with the time dependent temperature $T_{H}(t)$,

$$
D_{e}\left(T_{H}\right)=\frac{k_{B} T_{H}}{m \gamma_{e}\left(T_{H}\right)} .
$$

In the elastic limit, the $\lambda_{n}$ do not depend on time and define the modes of the system. There is a diffusive hydrodynamic mode given by $-D_{e} q^{2}$, which corresponds to $n_{i}=0, i=1, \cdots, d$, and an infinite set of kinetic modes, decaying much faster. The problem now is that the frequencies $\lambda_{n}$ depend on time and they do not characterize the time evolution of the system. This is a direct consequence of the dissipation in collisions. Nevertheless, it is possible to transform the Fokker-Planck equation into one with time independent coefficients by using dimensionless variables. This is similar to the transformations used in the two previous sections for the Navier-Stokes transport coefficients and for the self-diffusion equation. We introduce

$$
\tau=a\left(1-\epsilon_{0}\right) \int_{0}^{t} d t^{\prime} \gamma_{e}\left(t^{\prime}\right), \quad \boldsymbol{k}=\boldsymbol{q} \frac{\widehat{v}_{0}(t)}{a(\alpha)\left(1-\epsilon_{0}\right) \gamma_{e}\left(T_{H}\right)}, \quad \widehat{\boldsymbol{v}}=\frac{\boldsymbol{v}}{\widehat{v}_{0}(t)},
$$

where

$$
\widehat{v}_{0}^{2}=\frac{2 k_{B} T_{H}(t) a(\alpha)}{m\left(1-\epsilon_{0}\right)} .
$$

The Fokker-Planck equation becomes

$$
\left(\partial_{\tau}+i \boldsymbol{k} \cdot \widehat{\boldsymbol{v}}\right) \widehat{f}_{s}=\widehat{\mathcal{L}} \widehat{f}_{s} .
$$


Now the reduced density distribution is

$$
\widehat{f_{s}}(\boldsymbol{k}, \widehat{\boldsymbol{v}}, \tau)=\Omega \widehat{v}_{0}^{d}(t) f_{s}(\boldsymbol{q}, \boldsymbol{v}, t)
$$

and

$$
\widehat{\mathcal{L}} \equiv\left(1-\epsilon_{0}\right) \frac{\partial}{\partial \widehat{\boldsymbol{v}}} \cdot\left(\widehat{\boldsymbol{v}}+\frac{1}{2} \frac{\partial}{\partial \widehat{\boldsymbol{v}}}\right)
$$

The eigenvalues of the operator $\widehat{\mathcal{L}}+i \boldsymbol{k} \cdot \widehat{\boldsymbol{v}}$ are given by

where

$$
\widehat{\lambda}_{n}=-\left(1-\epsilon_{0}\right) \sum_{i=1}^{d} n_{i}-\frac{D_{e}^{*}}{\left(1-\epsilon_{0}\right)^{2}} k^{2}
$$

$$
D_{e}^{*}=\frac{1-\epsilon_{0}}{2}
$$

In this dimensionless form we easily identify kinetic modes and a diffusive mode. As compared with the elastic limit, in which $\epsilon_{0}=0$, the kinetic modes have been slowed by a factor $\left(1-\epsilon_{0}\right)$ while the diffusion mode has been enhanced by a factor $\left(1-\epsilon_{0}\right)^{-2}$. Nevertheless, the relevant qualitative picture of microscopic modes decaying faster then the macroscopic ones, and the long time evolution being described by the hydrodynamic mode is still valid. In other words, the "aging to hydrodynamics" also applies for the description of an inelastic Brownian particle in a low density granular flow.

The theory developed in this Section, i.e. the Brownian limit of the Boltzmann-Lorentz equation and the exact consequences we have derived from the Fokker-Planck equation (51), has also been confirmed on the basis of the DSMC method applied to the Boltzmann-Lorentz equation [31]. In particular, excellent agreement has been found for the approach to a homogeneous cooling state, the temperature of that state, the approach to diffusion as measured, for instance, by the mean square displacement, and the dependence of the diffusion coefficient on the coefficient of restitution. Since the discussion parallels in many points the presentation in the previous section, we do not repeat it here, and refer the reader to the literature for details.

\section{Conclusion}

We have presented several transport situations in which the transition from a kinetic regime to a hydrodynamic one has been verified by comparing the theoretical results coming from the hydrodynamic equations with the numerical solutions to the kinetic equations. Moreover, in one of the considered situations, self-diffusion, the results have also been shown to agree with Molecular Dynamics simulation data. The agreement extends in all cases over a wide range of values of dissipation and it is by no means restricted to the quasielastic limit. The Brownian motion problem is particularly relevant in this respect 
since it allows a detailed and exact study of the aging to hydrodynamics and the decay of kinetic or microscopic excitations.

The main physical consequence of the time dependence of the transport coefficients in the context of the validity of a hydrodynamic description, is the introduction of a new relevant time scale, implying that the decay of kinetic excitations in the original time scale $t$ is algebraical rather than exponential. This, in principle, is not inconsistent with a separation of time scales that is the condition required for a hydrodynamic description. In any case and in order to put the results presented in this paper in proper context, let us stress that we have considered values of the restitution coefficient larger than 0.6. It is then possible that for smaller $\alpha$ the hydrodynamic description and the own Boltzmann equation do not provide an accurate description of the evolution of the system.

The energy dissipation in collisions modifies the transport equations in both, a trivial and expected way and also in a subtle and hard to anticipate manner. The former is essentially given by the time dependence of the transport coefficients on time through the temperature and the presence of the energy source term in the equation for the temperature. Examples for the latter are the density gradient contribution to the heat flux and the modification of the fluctuation-dissipation relation in Brownian motion.

In this presentation many relevant aspects of rapid granular flows have not been addressed. In particular, all the discussion has been restricted to near the homogeneous cooling state situations and the stability of a freely evolving granular gas has not been considered. In spite of these limitations we believe it has been clearly established that the combination of analytical kinetic theory, direct simulation Monte Carlo methods, and Molecular Dynamics provides a unique way for approaching the study of rapid granular flows. As already mentioned, this possibility is not restricted to the very low density limit in which the Boltzmann equation applies, but can also be used in the context of the Enskog equation.

\section{Acknowledgements}

The material presented in this paper is based on published and unpublished work in collaboration with J. W. Dufty, M. J. Ruiz-Montero, A. Santos, F. Moreno, and R. García-Rojo, to whom I am greatly indebted. This research was partially supported by Grant No. PB98-1124 from the Dirección General de Investigación Científica y Técnica (Spain).

\section{References}

1. A classical and useful reference is: C. S. Campbell, Annu. Rev. Fluid Mech. 22, 57 (1990).

2. J. J. Brey, J. W. Dufty, and A. Santos, J. Stat. Phys. 87, 1051 (1997). 
3. C. K. K. Lun, S. B. Savage, D. J. Jeffrey, and N. Chepurniy, J. Fluid Mech. 140, 223 (1984).

4. J. T. Jenkins and M. W. Richman, Arch. Ration. Mech. Anal. 87, 355 (1985); Phys. Fluids 28, 3485 (1986).

5. N. Sela and I. Goldhirsch, J. Fluid Mech. 361, 41 (1998).

6. A. Goldshtein and M. Shapiro, J. Fluid Mech. 282, 75 (1995).

7. J. J. Brey, J. W. Dufty, C. S. Kim, and A. Santos, Phys. Rev. E 58, 4638 (1998); J. J. Brey and D. Cubero, unpublished.

8. V. Garzó and J. W. Dufty, Phys. Rev. E 59, 5895 (1999).

9. J. A. McLennan, Introduction to Nonequilibrium Statistical Mechanics (PrenticeHall, New Jersey, 1989).

10. P. Résibois and M. de Leener, Classical Kinetic Theory of Fluids, (John Wiley and Sons, New York, 1977).

11. H. Jaeger, S. Nagel, and R. Behringer, Rev. Mod. Phys. 68, 1250 (1996).

12. V. V. R. Natarajan, M. L. Hunt, and E. D. Taylor, J. Fluid Mech. 304, 1 (1995), and references therein.

13. O. Zik and J. Stavans, Europhys. Lett. 16, 255 (1991).

14. R. D. Wildman, J. M. Huntley, and J.-P. Hansen, Phys. Rev. E, 60, 7066 (1999).

15. C. S. Campbell, J. Fluid Mech. 348, 85 (1997).

16. R. F. Rodríguez, E. Salinas-Rodríguez. and J. W. Dufty, J. Stat. Phys. 32, 279 (1983).

17. J. J. Brey, J. W. Dufty, and A. Santos, J. Stat. Phys. 97, 281 (1999).

18. G. Bird, Molecular Gas Dynamics and the Direct Simulation of Gas Flows, (Clrendon Press, Oxford, 1994).

19. M. L. Tan and I. Goldhirsch, Phys. Rev. Lett. 81, 3022 (1998).

20. L. P. Kadanoff, Rev. Mod. Phys. 71, 435 (1999).

21. J. W. Dufty and J. J. Brey, Phys. Rev. Lett. 82, 4566 (1999).

22. J. J. Brey, M. J. Ruiz-Montero, and D. Cubero, Phys. Rev. E 54, 3664 (1996).

23. H. van Beijeren and M. H. Ernst, Physica A 68, 437 (1973); 70, 225 (1973).

24. J. J. Brey, M. J. Ruiz-Montero, and D. Cubero, Europhys. Lett. 48, 359 (1999)

25. D. M. Gass, J. Chem. Phys. 54, 1898 (1971).

26. P. Deltour and J. L. Barrat, J. Phys. I 7, 137 (1997).

27. J. J. Brey, M. J. Ruiz-Montero, D. Cubero, and R. García-Rojo, Phys. Fluids 12876 (2000).

28. T. P. C. van Noije and M. H. Ernst, Granular Matter 1, 57 (1998).

29. I. Goldhirsch and G. Zanetti, Phys. Rev. Lett. 70, 1619 (1993); S. McNamara and W. R. Young, Phys. Rev. E 50, R28 (1994).

30. H. Risken, The Fokker-Planck Equation (Springer-Verlag, Berlin, 1984).

31. J. J. Brey, M. J. Ruiz-Montero, D. Cubero, and J. W. Dufty, Phys. Rev. E 60, 7174 (1999). 